\title{
Duration of lipid peroxidation after acute spinal cord injury in rats and the effect of methylprednisolone
}

\author{
Laboratory investigation
}

\author{
Sean D. Christie, M.D., ${ }^{1,2}$ Ben Comeau, B.Sc., ${ }^{1}$ Tanya Myers, R.T., ${ }^{1}$ Damaso Sadi, B.Sc., ${ }^{1}$ \\ Mark Purdy, B.Sc., ${ }^{1}$ ANd Ivar Mendez, M.D., Ph.D. ${ }^{1,2}$
}

Departments of ${ }^{I}$ Anatomy and Neurobiology and ${ }^{2}$ Surgery (Neurosurgery), Dalhousie University, Halifax, Nova Scotia, Canada

\begin{abstract}
Object. Oxidative stress leading to lipid peroxidation is a major cause of secondary injury following spinal cord injury (SCI). The objectives of this study were to determine the duration of lipid peroxidation following acute SCI and the efficacy of short- and long-term administration of methylprednisolone on decreasing lipid peroxidation.

Methods. A total of 226 female Wistar rats underwent clip-compression induced SCI. In the first part of the study, spinal cords of untreated rats were assayed colorimetrically for malondialdehyde (MDA) to determine lipid peroxidation levels at various time points between 0 and 10 days. In the second part of the study, animals were treated with methylprednisolone for either 24 hours or 7 days. Control animals received equal volumes of normal saline. Treated and control rats were killed at various time points between 0 and 7 days.

Results. The MDA levels initially peaked 4 hours postinjury. By 12 hours, the MDA levels returned to baseline. A second increase was observed from 24 hours to 5 days. Both peak values differed statistically from the trough values $(\mathrm{p}<0.008)$. The methylprednisolone reduced MDA levels $(\mathrm{p}<0.04)$ within 12 hours of injury. No effect was seen at 24 hours or later.

Conclusions. The results of this study indicate that oxidative stress persists for 5 days following SCI in rats, and although methylprednisolone reduces MDA levels within the first 12 hours, it has no effect on the second lipid peroxidation peak. (DOI: 10.3171/FOC.2008.25.11.E5)
\end{abstract}

\section{KEY WORDS • lipid peroxidation • methylprednisolone • spinal cord injury $\quad \bullet \quad$ timing}

$\mathrm{S}$ PINAL cord injury has permanent and devastating effects on the lives of affected individuals. Major neurological deficits of SCI, such as paraplegia or tetraplegia, are the result of 2 closely related events, the primary mechanical injury and the subsequent secondary injury caused by biochemical reactions. ${ }^{2}$ Various mechanisms have been proposed to explain the cellular damage attributed to secondary injury. These include vascular, electrolyte, and biochemical changes as well as edema and loss of energy metabolism. ${ }^{44}$

Oxidative stress is a term frequently used to describe the numerous cytotoxic consequences of oxygen-free radicals and other chemically reactive species. These molecules are generated as by-products of normal and atypical metabolic processes that use molecular oxygen. ${ }^{13}$ Their reactivity is the result of an unpaired electron in their outer atomic orbital. ${ }^{8}$ It is the product of this reactivity that contributes to neuronal injury by reacting with

Abbreviations used in this paper: $\mathrm{MDA}=$ malondialdehyde; $\mathrm{SCI}=$ spinal cord injury; $\mathrm{SD}=$ standard deviation. endogenous proteins, ${ }^{1}$ lipids, and nucleic acids,${ }^{3}$ causing structural changes through reconfiguration and degradation. Oxidative stress initiates lipid peroxidation cascades that lead to the damage of highly vulnerable cell membranes during the first few days after injury. ${ }^{29}$ The detrimental effects of secondary injury have been shown to worsen over time (up to 3-6 weeks postinjury). ${ }^{44} \mathrm{Al}-$ though the pathological changes such as the development of a gliotic scar around the lesion, ${ }^{44}$ wallerian degeneration, axonal loss ${ }^{16}$ and prolonged ischemia ${ }^{2}$ are well characterized, the time course and magnitude of lipid peroxidation are not known beyond the initial 24 hours after the injury as the majority of studies on lipid peroxidation have measured markers only within the first 24 hours after injury. $4,7,21,39,42,43,45$ Establishing the duration of lipid peroxidation after the injury is important as reduction of lipid peroxidation with drugs such as methylprednisolone has been proposed as a potential therapeutic intervention in patients who have suffered SCI.

Methylprednisolone has been studied extensively in animal models of SCI. Early studies demonstrated a 
significant biochemical effect after the administration of high-dose methylprednisolone.$^{8-10,21-24,37}$ The potential for human application of this treatment led to the development of the National Acute Spinal Cord Injury Studies.5,6 The proposed mechanism of action of methylprednisolone has been the reduction of secondary injury by scavenging lipid peroxyl radicals, thereby inhibiting the lipid peroxidation cascade. ${ }^{17,18,22,23,25,43}$ This in turn has been thought to result in preservation of neurons, axons, myelin, and intracellular organelles, including the mitochondria and nucleus..$^{30,31}$ However, more recent studies have suggested that methylprednisolone acts preferentially on glia and has less of an effect on neurons, ${ }^{33}$ and seems to have a significant impact on glial activation, thereby reducing the production of inhibitory chondroitin sulfate proteoglycans $\mathrm{s}^{36}$ and possibly facilitating an environment more suitable for regeneration.

This study was designed to determine the levels and the duration of lipid peroxidation after the initial injury in a rat model of SCI caused by clip compression and the effectiveness of methylprednisolone in reducing this lipid peroxidation.

\section{Methods}

The following protocol was reviewed and approved by the local University Animal Care Committee, and all procedures performed throughout this investigation were conducted in accordance with the guidelines of the Canadian Council of Animal Care and the University Council on Laboratory Animals.

\section{Animal Preparation}

A total of 266 female Wistar rats (250-300 g, Charles River) were used. The animals were housed 2 per cage prior to surgery with food and water ad libitum. After arrival in our animal care facility, the animals were allowed to acclimatize for a minimum of 3 days prior to surgery. After surgery, the animals were housed individually to prevent injury caused by their cage mates, and they were monitored closely for illness.

\section{Surgery and Postoperative Care}

The animals were anesthetized using a $2.0-\mathrm{ml} / \mathrm{kg}$ intraperitoneal injection of a mixture of $25 \%$ ketamine hydrochloride (Ketalean, MTC Pharmaceuticals), 6\% xylazine (Rompun, Miles Canada), and 2.5\% acepromazine maleate (Wyeth-Ayerst Canada) in 0.9\% saline. Betadine surgical scrub (7.5\% povidone-iodine, Purdue Frederick, Inc.) was used for sterile preparation of the surgical field after shaving. A dissecting microscope facilitated exposure and laminectomies at the T9-10 level. A severe SCI was induced at T-10 via a clip compression (60 $\mathrm{g}$ for 1 minute). The wound was irrigated and the muscle and skin closed in layers with 000 polyglactin suture (Ethicon).

Animals were provided soft bedding and fresh hay. Wooden blocks were placed in each cage to serve as distractions to reduce autotomy. Intraoperative and postoperative body temperatures were maintained using a
TABLE 1: Stratification of 226 rats among the 3 experimental groups*

\begin{tabular}{|c|c|c|c|c|c|}
\hline \multirow{3}{*}{$\begin{array}{c}\text { Expected } \\
\text { Postinjury } \\
\text { Survival } \\
\text { (hrs) }\end{array}$} & \multirow[b]{3}{*}{ Duration } & \multicolumn{4}{|c|}{ Experimental Group (no. of rats) } \\
\hline & & \multicolumn{2}{|c|}{$24-\mathrm{Hr}$} & \multicolumn{2}{|c|}{$168-\mathrm{Hr}$} \\
\hline & & MP & NS & MP & NS \\
\hline 0 & $6 \dagger$ & 5 & 5 & 0 & 0 \\
\hline 1 & 4 & 5 & 5 & 0 & 0 \\
\hline 4 & 4 & 5 & 5 & 0 & 0 \\
\hline 6 & 4 & 0 & 0 & 6 & 6 \\
\hline 12 & 5 & 5 & 5 & 6 & 6 \\
\hline 18 & 5 & 0 & 0 & 0 & 0 \\
\hline 24 & 7 & 5 & 5 & 6 & 6 \\
\hline 36 & 5 & 0 & 0 & 0 & 0 \\
\hline 48 & 6 & 5 & 5 & 6 & 6 \\
\hline 72 & 8 & 0 & 0 & 0 & 0 \\
\hline 96 & 5 & 0 & 0 & 0 & 0 \\
\hline 120 & 5 & 5 & 5 & 6 & 6 \\
\hline 144 & 4 & 0 & 0 & 0 & 0 \\
\hline 168 & 8 & 0 & 0 & 6 & 6 \\
\hline 240 & 8 & 0 & 0 & 0 & 0 \\
\hline total & 84 & 35 & 35 & 36 & 36 \\
\hline
\end{tabular}

* MP = methylprednisolone; NS = normal saline.

$\dagger$ These animals served as a control for the lipid peroxidation assay and did not undergo lesioning.

heating pad. Softened food was prepared twice daily and placed on the cage floor within reach of the animals. The rats were washed and had their bladders expressed twice daily for the duration of the experiment. All animals were monitored for urinary tract infections and when present, were treated with $10 \%$ enrofloxacin $(1.0 \mathrm{ml} / \mathrm{kg}$ subcutaneous daily; Baytril, Bayer, Inc.).

\section{Study Design and Treatments}

This study consisted of the following 3 experiments (Table 1): 1) an investigation of the time course of lipid peroxidation following acute SCI (duration group; 84 rats); 2) short-term treatment (24-hour group; 70 rats [35 each receiving methylprednisolone or normal saline]); and 3) long-term treatment (168-hour [7-day] group; 72 rats [36 each receiving methylprednisolone or normal saline).

Six animals in the duration group served as controls for the lipid peroxidation assay and did not undergo lesioning. The animals in the duration experiment were naive to any treatment of their SCI.

Based on previously reported studies,,$^{24,32,35,40,41}$ the animals treated with methylprednisolone received an initial $30-\mathrm{mg} / \mathrm{kg}$ bolus intraperitoneally, followed by an infusion of $5.4 \mathrm{mg} / \mathrm{kg} / \mathrm{hr}$. Control animals received equal volume injections of vehicle (normal saline). The animals in the 24-hour group received intraperitoneal injections for a total of 24 hours. The animals in the 168-hour group received an intraperitoneal infusion of methylprednisolone for a total of 7 days, administered via implanted os- 
Time course of lipid peroxidation after SCI

TABLE 2: Mean wet spinal cord weights prior to MDA assay*

\begin{tabular}{lc}
\hline \multicolumn{1}{c}{ Experiment } & Mean Spinal Cord Weight in g ( \pm SD) \\
\hline duration group & $0.53 \pm 0.06$ \\
24-hr group & \\
MP-treated & $0.48 \pm 0.08$ \\
NS-treated & $0.53 \pm 0.06$ \\
168-hr group & \\
MP-treated & $0.49 \pm 0.04$ \\
NS-treated & $0.50 \pm 0.06$ \\
all animals & $0.51 \pm 0.06$ \\
\hline
\end{tabular}

* There were no significant differences between any of the groups.

motic pumps (Alzet). The time points selected for death in both groups were matched to time points used in the Duration experiment.

At the appropriate time the animals were killed using an overdose of anesthetic and perfused transcardially with $300 \mathrm{ml}$ of isotonic saline. The entire spinal cord was quickly dissected and weighed. A tissue homogenate was made in $20 \mathrm{mM}$ phosphate buffer $\mathrm{pH} 7.4(0.40-0.60 \mathrm{~g}$ tissue $/ 2 \mathrm{ml}$ buffer), containing $20 \mu \mathrm{l}$ of $5 \mathrm{mM}$ butylated hydroxytoluene in acetonitrile. The homogenate was centrifuged to remove large particles and kept on ice.

\section{Tissue Analysis}

A Lowry assay was performed to ensure that the supernatant of the homogenate contained $15-60 \mathrm{mg} / \mathrm{ml}$ protein, the level necessary for the assay. Two 200- $\mu 1$ samples of the homogenate were each added to $800 \mu \mathrm{l}$ of water and $5.0 \mathrm{ml}$ of Working Lowry Solution $\left(2 \% \mathrm{Na}_{2} \mathrm{CO}_{3}\right.$ in $0.1 \mathrm{~N} \mathrm{NaOH}, 1 \% \mathrm{CuSO}_{4}, 0.5 \%$ potassium tartrate). This solution was incubated at room temperature for $25 \mathrm{~min}$ utes. After incubation, $400 \mu \mathrm{l}$ of Folin-Ciocalteu reagent (Sigma Aldrich) was added to each sample incubated at room temperature for an additional 30 minutes. The optical density for each sample was measured at $750 \mathrm{~nm}$ with reference to distilled water.

A colorimetric assay (Oxford Biomedical Research) was used as an indicator for lipid peroxidation in the tissue by measuring the MDA concentrations. Four 200- $\mu 1$ aliquots of supernatant from the centrifuged tissue homogenate for each animal were placed in microcentrifuge tubes. One aliquot from each animal was used as a "sam-

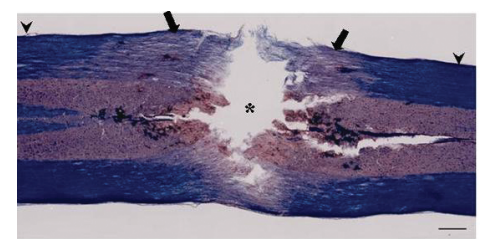

FIG. 1. Photomicrograph showing horizontal section of rat spinal cord 24 hours after injury showing a typical clip compression injury. This section depicts cavitation and necrosis (asterisk) within the gray matter and reduced myelination (arrows) within the white matter close to the lesion. The arrowheads indicate a more normal pattern of staining. Luxol fast blue (outer area), $\mathrm{H} \& \mathrm{E}$ (inner area). Scale bar $=500 \mu \mathrm{m}$.

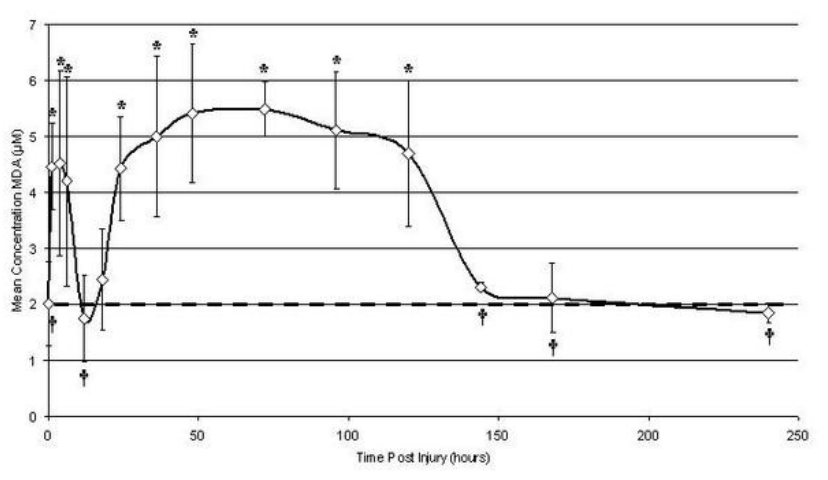

FIG. 2. Graph showing the concentration of MDA \pm SD as a function of time postinjury. The values denoted with the asterisk are significantly different from those denoted by the cross $(p<0.008)$. The dashed line represents the baseline mean value of the nonlesioned rats.

ple blank" and mixed with $650 \mu$ l of acetonitrile (75\%) in methanol (25\%). The other samples were mixed with $650 \mu \mathrm{l}$ of $N$-methyl-2-phenylindole and $150 \mu \mathrm{l}$ of $12-\mathrm{M}$ $\mathrm{HCl}$. All samples were then incubated at $45^{\circ} \mathrm{C}$ for $60 \mathrm{~min}$ utes. The resulting solutions were centrifuged at 10,000 $\mathrm{G}$ for 10 minutes. The supernatants were transferred to cuvettes, and the absorbance was recorded at $586 \mathrm{~nm}$. The concentration of MDA for each sample was then calculated by reference to a standard curve. The concentration of MDA for each animal was recorded as the mean value of the 3 samples for that animal.

\section{Statistical Analysis}

Within each experiment the MDA values were compared using a one-way analysis of variance - the time postlesion as the independent variable and MDA concentration as the dependent variable. Significant differences were examined using the Tukey post hoc test. Reported probability values reflect the results of the post hoc tests. Individual group means for death and weight loss were compared using a t-test.

\section{Results}

\section{Extent of Injury}

All lesioned animals had complete loss of motor control and bladder function below the level of injury. This persisted for the duration of the experiment. Histologically the injured region of the spinal cord demonstrated central necrosis with cavitation and a surrounding inflammatory infiltrate. Regions of intact neural tissue displayed a reduction in myelin staining indicative of wallerian degeneration. Figure 1 illustrates the typical histological appearance at 24 hours after clip compression.

\section{Spinal Cord Weights and Lowry Assay}

The mean wet weight of the spinal cords from all groups was $0.51 \pm 0.06 \mathrm{~g}( \pm \mathrm{SD})$. The mean weights for each group in each experiment are listed in Table 2. There was no statistical difference in mean weights between any of the groups examined. The protein concentrations cal- 


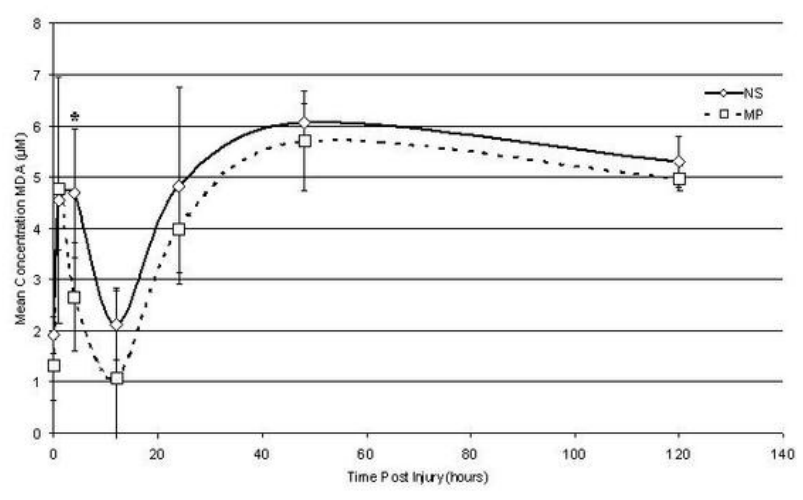

FIG. 3. Graph showing the mean concentration of MDA in the 24hour methylprednisolone (MP)-treated animals compared with that in the normal saline (NS) controls. Whiskers indicate the SD. ${ }^{*} p<0.04$.

culated using the Lowry assay showed that the supernatants of the homogenates contained between 20 and 50 $\mathrm{mg}$ protein/ml, which is within the suggested limits for accuracy of the MDA assay.

\section{Lipid Peroxidation Levels}

The MDA concentrations are reported as means \pm SD. The baseline MDA concentration in nonlesioned animals was $2.00 \pm 0.75 \mu \mathrm{M}$. After acute SCI the levels rose quickly to $4.52 \pm 1.65 \mu \mathrm{M}$ at 4 hours. This peak was short-lived as the MDA level returned to baseline at 12 hours $(1.74 \pm 0.78 \mu \mathrm{M})$. A second increase in MDA level was observed by 24 hours $(4.43 \pm 0.92 \mu \mathrm{M})$ and was sustained for 120 hours $(4.70 \pm 1.31 \mu \mathrm{M})$ before returning to baseline at 144 hours $(2.30 \pm 0.072 \mu \mathrm{M})$. The difference between the 2 peaks and the baseline level was statistically significant ( $\mathrm{p}<0.008$; Fig. 2).

\section{Methylprednisolone Treatment (24-Hour Group)}

The methylprednisolone-treated animals and normal saline controls exhibited a sharp increase in MDA levels after injury $(4.78 \pm 1.24$ and $4.55 \pm 2.44 \mu \mathrm{M}$ at 1 hour,

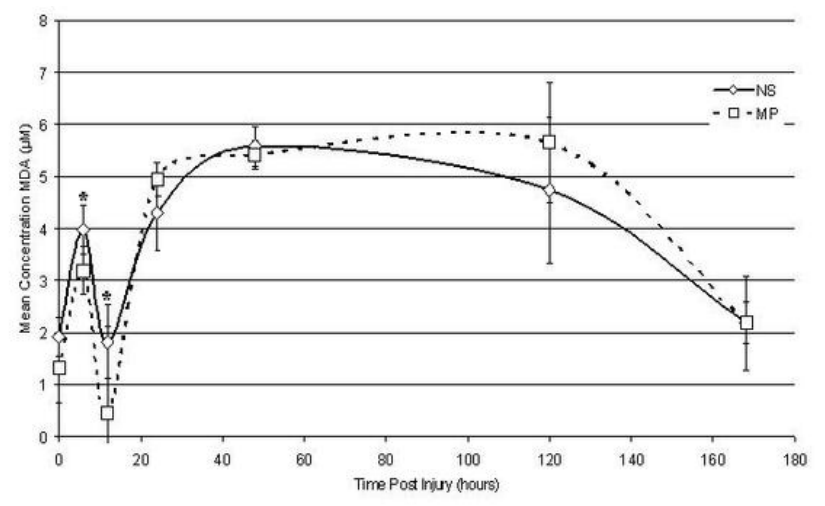

FIG. 4. Graph showing the mean concentration of MDA in the 7-day methylprednisolone-treated animals compared with that in normal saline controls. Whiskers indicate SD in relation to time. ${ }^{*} p<0.01$.

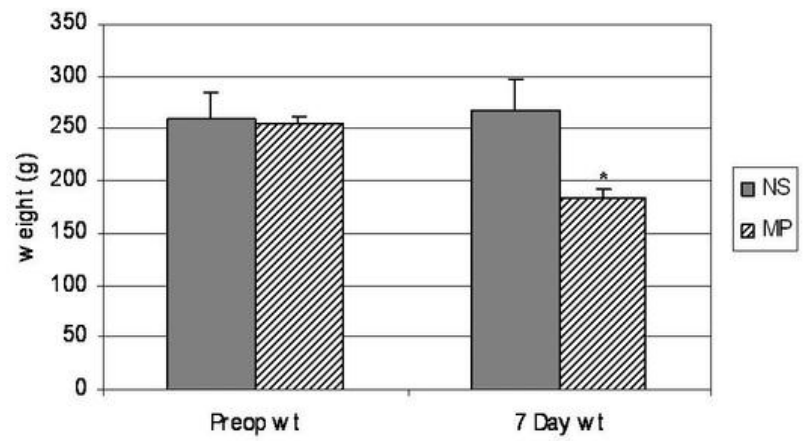

FIG. 5. Graph showing the difference in weight (wt) loss at 7 days between the methylprednisolone-treated animals and the normal saline controls. ${ }^{*} p<0.0001$.

respectively). These values were not statistically different. At 4 hours, however, the methylprednisolone group displayed significantly lower MDA levels than those in the normal saline group $(2.66 \pm 1.06$ vs $4.68 \pm 1.24 \mu \mathrm{M}$, p $<0.04)$. Both groups displayed a similar decrease in MDA levels at 12 hours followed by another increase at 24 hours. There was no statistical difference between the methylprednisolone and normal saline groups at 12 hours and later (Fig. 3). No rats died in either group.

\section{Methylprednisolone Treatment (168-Hour Group)}

The animals treated with methylprednisolone displayed a statistically significant reduction in the concentration of MDA at 6 and 12 hours after injury when compared with normal saline controls $(3.19 \pm 0.46 \mu \mathrm{M}$ and $0.46 \pm 1.64 \mu \mathrm{M}$ [for the methylprednisolone group] vs $3.98 \pm 0.46 \mu \mathrm{M}$ and $1.82 \pm 0.70 \mu \mathrm{M}$ [for the normal saline group], $\mathrm{p}<0.01)$. Both groups exhibited a second increase in MDA concentration by 24 hours, which persisted for 120 hours before returning to baseline. There was no statistical difference between the methylprednisolone and normal saline groups after 12 hours (Fig. 4).

The animals in both groups examined at the latest time point (168 hours) had initially experienced equal weight loss. However, at 7 days the animals treated with methylprednisolone had a persistent average weight loss of $28.0 \%$ compared with those in the normal saline control group, which averaged a $6.4 \%$ weight gain from their preoperative measurements ( $\mathrm{p}<0.0001$; Fig. 5).

In addition, the methylprednisolone-treated animals in this experiment were the only ones that sustained any predetermined mortality. Of the animals expected to survive 120 and 168 hours, 3 (25\%) of 12 died prematurely. One died at 4 days of a complicated urinary tract infection. The remaining 2 were killed at 5 and 6 days at the request of the animal care facility because of their weight loss. There was no statistical difference in mortality rates between the treated animals and controls in this experiment when comparing both groups as a whole (3 of 36 vs 0 of 36 rats, respectively; $p=0.62$ ) or only the animals assigned to the 2 longest time points, 120 and 168 hours ( 3 of 12 vs 0 of 12 rats, respectively; $p=0.39$ ). 


\section{Discussion}

An effective treatment strategy to reduce or reverse the permanent effects of SCI remains elusive. Understanding the complexity of biochemical and structural changes that occur following acute injury may be a key to developing effective therapies in the future.

Lipid peroxidation is one of the most important and damaging effects of free radicals following SCI and a key mechanism in oxidative stress. ${ }^{2,3,19,20,25}$ The oxidation of membrane lipids increases the permeability and fluidity of the membrane to ions, decreases numerous membrane adenosine triphosphatase activities, ${ }^{28}$ and increases rigidity.$^{14}$ Normal cellular membrane functioning is closely related to the presence of unsaturated and polysaturated lipid side chains. ${ }^{14}$ The cascade of events culminating in lipid peroxidation begins with the interaction between a free radical and the phospholipid bilayer of the cellular membrane. A hydrogen atom is removed from a methylene carbon of a polyunsaturated fatty acid $^{28}$ resulting in the creation of a lipid radical. This newly formed radical can then interact with molecular oxygen to produce a peroxyl radical. At this point, a self-propelling chain of further lipid peroxidation can be propagated by the removal of a methylene carbon from another polyunsaturated fatty acid. Repetition of this process greatly alters the properties of the cell membrane including its fluidity, thereby affecting function and cell survival. ${ }^{14}$

The present study has demonstrated that acute oxidative stress, as measured by a lipid peroxidation marker (MDA), persists for up to 120 hours (5 days) after acute SCI in rats. This observation strongly suggests that lipid peroxidation with its deleterious effects are present for much longer than the 48 hours as previously described. ${ }^{31}$ The most striking finding in the time course of lipid peroxidation was the presence of 2 separate, and statistically distinct, peaks from 1 to 6 hours and from 24 to 120 hours postinjury (Fig. 2). Qian and Liu ${ }^{39}$ reported a similar early increase in MDA levels followed by a return toward baseline at 9 hours after injury. However, in that report the authors did not examine MDA levels beyond that time point.

The cause of the 2 observed MDA peaks is unclear. Elevated levels will result from damage to both gray and white matter as well as from both primary and secondary injury mechanisms. However, the precise mechanisms and anatomical locations of these events cannot be determined in this study. Nevertheless, it is likely that each lipid peroxidation peak (Fig. 2) represents the summation of the various biochemical events occurring at that particular point in time. The observed time course of these events may help us postulate the underlying cause of the lipid peroxidation at various time points after SCI. For example, glutamate excitotoxicity is believed to manifest rapidly after injury, toxic levels detected by 15 minutes and peak levels at 6 hours, $3,12,26,27$ and may contribute to the initial increase seen within the first 12 hours (Fig. 2). Ischemia also occurs within the 1st hour after injury and persists for at least 24 hours. ${ }^{44}$ Infiltration of neutrophils into the site of injury has been shown to occur by 6 hours and is followed by activated macrophages/micro- glia, which peak between 24 and 48 hours after injury and persist during the 1st week..$^{11,26,38}$ These events may interact with each other to produce the temporal variations in MDA production that we have observed during the 1st week after injury. In this regard, developing treatments that reduce lipid peroxidation targeted at different mechanisms may be of great benefit in reducing secondary injury during the 1st week after SCI.

Methylprednisolone has been previously shown to reduce MDA levels in experimental studies, providing evidence of functional improvement compared with controls. ${ }^{23}$ These measurements were primarily taken within the first few hours after injury ${ }^{21-23,45}$ and correlate well with the reduction in levels we observed in the first peak. However, neither short-term (24-hour) nor continuous infusion of methylprednisolone had any effect on the second MDA peak observed in this study. The lack of effect may have been due to a subtherapeutic dosage of drug or that the predominant underlying pathological process is not amenable to methylprednisolone therapy. The routine use of methylprednisolone in clinical practice has come under scrutiny in recent years. Magnetic resonance imaging findings in humans have suggested that only a minimal gross anatomical effect is observed in those treated with methylprednisolone. ${ }^{34}$ Furthermore there is growing evidence that methylprednisolone may induce a negative interaction with other therapies, such as erythropoietin ${ }^{15}$ and anti-CD11c monoclonal antibody ${ }^{46}$ which exert a positive effect when administered on their own. Therefore, the relative biochemical impact of this therapy must be kept in mind when developing future strategies.

\section{Conclusions}

In this study we have established that oxidative stress, as measured by MDA levels, persists for up to 5 days after acute SCI in rats. Methylprednisolone reduces the concentration of MDA within the first 12 hours after injury. Neither short-term (24 hours) nor long-term (7 days) administration of methylprednisolone showed any effect on the second, more prolonged MDA peak, which lasts from 24 hours to 5 days. The weight loss and mortality rates were greater in the 7-day methylprednisolone group. This novel observation of sustained levels of oxidative stress may have important implications on the development of future strategies to treat the secondary injury after an acute SCI.

\section{Disclosure}

Financial support for this study was provided by the Canadian Institutes of Health Research, Nova Scotia Health Research Foundation, and the Capital Health Research Fund (to Dr. Christie).

\section{References}

1. Aksenova M, Butterfield D, Zhang S, Underwood M, Geddes $\mathrm{J}$ : Increased protein oxidation and decreased creatine kinase BB expression and activity after spinal cord contusion injury. J Neurotrauma 19:491-502, 2002

2. Amar A, Levy M: Pathogenesis and pharmacological strategies for mitigating secondary damage in acute spinal cord injury. Neurosurgery 44:1027-1039, 1999 


\section{S. D. Christie et al.}

3. Azbill R, Mu X, Bruce-Keller A, Mattson M, Springer J: Impaired mitochondrial function, oxidative stress and altered antioxidant enzyme activities following traumatic spinal cord injury. Brain Res 765:283-290, 1997

4. Barut S, Canbolat A, Bilge T, Aydin Y, Cokneseli B, Kaya U: Lipid peroxidation in experimental spinal injury: time-level relationship. Neurosurg Rev 16:53-59, 1993

5. Bracken MB, Shepard MJ, Collins WF, Holford TR, Young W, Baskin DS, et al: A randomized, controlled trial of methylprednisolone or naloxone in the treatment of acute spinal-cord injury. Results of the Second National Acute Spinal Cord Injury Study. N Engl J Med 322:1405-1411, 1990

6. Bracken MB, Shepard MJ, Holford TR, Leo-Summers L, Aldrich EF, Fazl M, et al: Administration of methylprednisolone for 24 or 48 hours or trilazad mesylate for 48 hours in the treatment of acute spinal cord injury. JAMA 277:1597-1604, 1997

7. Braughler JM: Lipid peroxidation-induced inhibition of gamma-aminobutyric acid uptake in rat brain synaptosomes: Protection by glucocorticoids. J Neurochem 44:1282-1288, 1985

8. Braughler JM, Hall ED: Involvement of lipid peroxidation in CNS injury. J Neurotrauma 9 (1 Suppl):S1-S7, 1992

9. Braughler JM, Hall ED: Lactate and pyruvate metabolism in injured cat spinal cord before and after a single large intravenous dose of methylprednisolone. J Neurosurg 59:256-261, 1983

10. Braughler JM, Hall ED, Means ED, Waters TR, Anderson DK: Evaluation of an intensive methylprednisolone sodium succinate dosing regimen in experimental spinal cord injury. J Neurosurg 67:102-105, 1987

11. Carlson SL, Parrish ME, Springer JE, Doty K, Dossett L: Acute inflammatory response in spinal cord following impact injury. Exp Neurol 151:77-88, 1998

12. Chu G, Tator C, Tyminanski M: Calcium and neuronal death in spinal neurons, in Kalb R, Strittmatter S (eds): Neurobiology of Spinal Cord Injury. New Jersey: Humana Press, 2000, pp 23-56.

13. Coyle J, Puttfarcken P: Oxidative stress, glutamate, and neurodegenerative disorders. Science 262:689-694, 1993

14. Farooqui A, Horrocks L: Plasmalogens: workhorse lipids of membranes in normal and injured neurons and glia. Neuroscientist 7:232-245, 2001

15. Gorio A, Madaschi L, Di Stefano B, Carelli S, Di Giulio AM, De Biasi S, et al: Methylprednisolone neutralizes the beneficial effects of erythropoietin in experimental spinal cord injury. Proc Natl Acad Sci U S A 102:16379-16384, 2005

16. Haghighi S, Agrawal SK, Surdell D, Plambeck R, Agrawal S, Johnson G, et al: Effects of methylprednisolone and MK-801 on functional recovery after experimental chronic spinal cord injury. Spinal Cord 38:733-740, 2000

17. Hall ED: Inhibition of lipid peroxidation in CNS trauma. $\mathbf{J}$ Neurotrauma 8 (1 Suppl):S31-S40, 1991

18. Hall ED: The neuroprotective pharmacology of methylprednisolone. J Neurosurg 76:13-22, 1992

19. Hall ED: Pharmacological treatment of acute spinal cord injury: how do we build on past success? J Spinal Cord Med 24:142-146, 2001

20. Hall ED: The role of oxygen radicals in traumatic injury: clinical implications. J Emerg Med 11 (suppl 1):31-36, 1993

21. Hall ED, Braughler JM: Acute effects of intravenous glucocorticoid pretreatment on the in vitro peroxidation of cat spinal cord tissue. Exp Neurol 73:321-324, 1981

22. Hall ED, Braughler JM: Effects of intravenous methylprednisolone on spinal cord lipid peroxidation and $\mathrm{Na}++\mathrm{K}+$ )ATPase activity. Dose-response analysis during 1st hour after contusion injury in the cat. J Neurosurg 57:247-253, 1982

23. Hall ED, Braughler JM: Glucocorticoid mechanisms in acute spinal cord injury: a review and therapeutic rationale. Surg Neurol 18:320-327, 1982

24. Hall ED, Wolf DL, Braughler JM: Effects of a single large dose of methylprednisolone sodium succinate on experimental posttraumatic spinal cord ischemia. Dose-response and time-action analysis. J Neurosurg 61:124-130, 1984

25. Hall ED, Yonkers PA, Andrus PK, Cox JW, Anderson DK: Biochemistry and pharmacology of lipid antioxidants in acute brain and spinal cord injury. J Neurotrauma 9 (2 Suppl): S25-S442, 1992

26. Hausmann ON: Post-traumatic inflammation following spinal cord injury. Spinal Cord 41:369-378, 2003

27. Hulsebosch CE: Recent advances in pathophysiology and treatment of spinal cord injury. Adv Physiol Educ 26:238255,2002

28. Juurlink B, Patterson P: Review of oxidative stress in brain and spinal cord injury: suggestions for pharmacological and nutritional management strategies. J Spinal Cord Med 21:309-330, 1998

29. Kamencic H, Griebel RW, Lyon AW, Paterson PG, Juurlink $\mathrm{BH}$ : Promoting glutathione synthesis after spinal cord trauma decreases secondary damage and promotes retention of function. FASEB J 15:243-250, 2001

30. Kaptanoglu E, Caner H, Surucu H, Akbiyik F: Effect of mexiletine on lipid peroxidation and early ultrastructure findings in experimental spinal cord injury. J Neurosurg Spine 91:200-204, 1999

31. Kaptanoglu E, Tuncel M, Palaoglu S, Konan A, Demirpence E, Kilinc K: Comparison of the effects of melatonin and methylprednisolone in experimental spinal cord injury. J Neurosurg (2 Suppl) 9:77-84, 2000

32. Koc RK, Akdemir H, Karakucuk EI, Oktem IS, Menku A: Effect of methylprednisolone, tirilazad mesylate and vitamin E on lipid peroxidation after experimental spinal cord injury. Spinal Cord 37:29-32, 1999

33. Lee JM, Yan P, Xiao Q, Chen S, Lee KY, Hsu CY, et al: Methylprednisolone predicts oligodendrocytes but not neurons after spinal cord injury. J Neurosci 28:3141-3149, 2008

34. Leypold BG, Flanders AE, Schwartz ED, Burns AS: The impact of methylprednisolone on lesion severity following spinal cord injury. Spine 32:373-378, 2007

35. Liu D, Li L, Augustus L: Prostaglandin release by spinal cord injury mediates production of hydroxyl radical, malondialdehyde and cell death: a site of the neuroprotective action of methylprednisolone. J Neurochem 77:1036-1047, 2001

36. Liu WL, Lee YH, Tsai SY, Hsu CY, Sun YY, Yang LY, et al: Methylprednisolone inhibits the expression of glial fibrillary acidic protein and chondroitin sulphate proteoglycans in reactivated astrocytes. Glia 56:1390-1400, 2008

37. Means ED, Anderson DK, Waters TR, Kalaf L: Effect of methylprednisolone in compression trauma to the feline spinal cord. J Neurosurg 55:200-208, 1981

38. Popovich PG, Wei P, Stokes BT: Cellular inflammatory response after spinal cord injury in Sprague-Dawley and Lewis rats. J Comp Neurol 377:433-464, 1997

39. Qian H, Liu D: The time course of malondialdehyde production following impact injury to rat spinal cord as measured by microdialysis and high pressure liquid chromatography. Neurochem Res 22:1231-1236, 1997

40. Rabchevsky AG, Fugaccua I, Sullivan PG, Blades DA, Scheff SW: Efficacy of methylprednisolone therapy for the injured rat spinal cord. J Neurosci Res 68:7-18, 2002

41. Ross IB, Tator CH: Spinal cord blood flow and evoked potential responses after treatment with nimodipine or methylprednisolone in spinal cord-injured rats. Neurosurgery 33:470-476, 1993

42. Springer JE, Azbill RD, Mark RJ, Begley J, Waeg G, Mattson MP: 4-hydroxynonenal, a lipid peroxidation product, rapidly 


\section{Time course of lipid peroxidation after SCI}

accumulates following traumatic spinal cord injury and inhibits glutamate uptake. J Neurochem 68:2469-2476, 1997

43. Taoka Y, Okajima K, Uchiba M, Johno M: Methylprednisolone reduces spinal cord injury in rats without affecting tumor necrosis factor-alpha production. J Neurotrauma 18:533543,2001

44. Tator CH, Fehlings MG: Review of the secondary injury theory of acute spinal cord trauma with emphasis on vascular mechanisms. J Neurosurg 75:15-26, 1991

45. Topsakal C, Erol FS, Ozveren MF, Yilmaz N, Ilhan N: Effects of methylprednisolone and dextromethorphan on lipid peroxidation in an experimental model of spinal cord injury. Neurosurg Rev 25:258-266, 2002
46. Weaver LC, Gris D, Saville LR, Oatway MA, Chen Y, Marsh DR, et al: Methylprednisolone causes minimal improvement after spinal cord injury in rats, contrasting with benefits of an anti-integrin therapy. J Neurotrauma 22:1375-1387, 2005

Manuscript submitted July 14, 2008.

Accepted September 9, 2008.

Address correspondence to: Sean D. Christie, M.D., Department of Surgery (Neurosurgery), Dalhousie University, 3814-1796 Summer Street, Halifax, Nova Scotia, Canada B3H 3A7. email: sean.christie@dal.ca. 